First publ. in: Annalen der Physik, 8. Serie, 6 (1997), 6, pp. 425-447

\title{
Hydrogen and helium films as model systems of wetting*
}

\author{
S. Herminghaus ${ }^{1}$ ), J. Vorberg ${ }^{1}$ ), H. Gau ${ }^{1}$ ), R. Conradt ${ }^{2}$ ), D. Reinelt ${ }^{2}$ ), \\ H. Ulmer ${ }^{2}$ ), P. Leiderer ${ }^{2}$ ), and M. Proyrembel ${ }^{3}$ ) \\ I) Max Planck Institut für Kolloid und Grenzllichenforsehung, Rudower Chatussee 5. \\ D-12489 Berlin-Adlershof, Germany; sherming @mpikg fa-berlinde \\ 2) Universitil Konstanz, Fakultii fur Physik, Unwersiatsstrasse 10, D 78434 Konstanz, Gemany; \\ paul.Jeiderer@uni-konstanz.de \\ Universität Mainz, Institut für Physik, Staudinger Weg 7, D-55099 Mainz, Germany
}

Received 23 February 1997, accepted 17 March 1997

\begin{abstract}
Optical experiments on the wetting properties of liquid ${ }^{4} \mathrm{He}$ and molecular hydrogen are reviewed. Hydrogen films on noble netal surfaces serve as model systenis for studying triple point wetting, a continuous transition between wetting and non-wetting. By means of optically excited surface plasmons, the adsorbed film thickness for temperatures around, and far below, the bulk melting temperature is measured, and the physical mechanisms responsible for the transition ato alucidated. Possible applications for other experiments in pure and applied research are discussed. Thin films and droplets of liquid helium are studied on cesium surfaces, on which there is a first order wetting transition. Our studies concentrate on dynamical observations via surface plasmon microscopy, which provide insight into the morpholngy of liquid helium droplets spreading at different temperatures. Features corresponding to pinning forces, the prewetting line, and the Kosterliz-Thotless transition arc clearly observed.
\end{abstract}

Keywords: Wetting; Surface plasmon; Quantum liquids

\section{Introduction}

The fascinating field of welting phenomena may be defined by the question: "under what circumstances, and in which morphology, is a material, $M_{1}$, thermodynamically stable at the boundary between two other given materials, $\mathrm{M}_{2}$ and $\mathrm{M}_{3}$ ?' ${ }^{1}$. This question is obviously a key issue in contemporary natural sciences as well as in many ficlds of moden technology, which have all concentrated more and more on systems in which interfaces and thin films play an important role. The need of understanding the behaviour of systems whose properties and performance are strongly influenced by interface phenomena entails the necessity of understanding wetling phenomena in general.

\footnotetext{
* Editorial note: This review anticle was received by $\mathrm{K}$. Dransfeld.

In mariy cases, $M_{2}$ denotes a gas, such as the gas phase of $M_{1}$, but this does not need to be the case.
} 


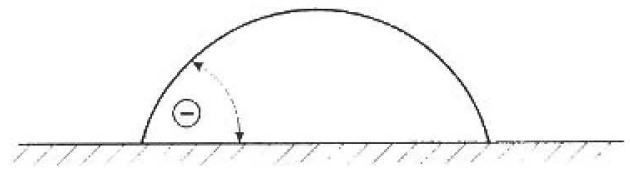

(b)

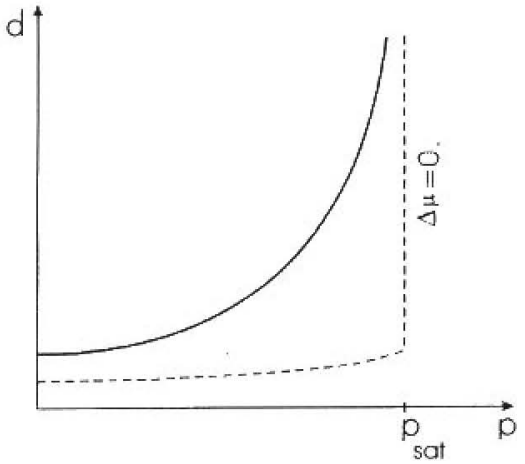

Fig. 1 Paradigms of wetting: a) contict angle 0 . b) thickness of adsorbed layer when coexistence is approached at fixed temperature (adsorption isotherm). Solid line: complete wetting. Dastacd line: incomplete wetting.

In spite of the fact that welting has been systematically investigated for almost two centuries $[1-3]$, there are still many unsolved problems and open questions to address. The particular challenge for both theoretical and experimental investigations is that macroscopic wetling properties may be changed dramatically by only minute changes in the microscopic properties of the involved materials and their surfaces. For example, a single layer of alcane molecules on a mica surface may change its wetting behaviour from perfectly hydrophilic to hydrophobic. The most striking experimental significance of this fact is the sensitivity of wetting phenomena to extraordinarily small amounts of surface contamination [4].

More specifically, the wetting properties of a substrate/adsorbite system are determined on the microscopic level by both shot and long range forces. The most prominent (and ubiquitous) long range force involved is the van der Waals force, which is of electromagnetic origin and contributes to the chemical potential of the adsorbed molecules as

$$
\Delta / t=\frac{\alpha}{d^{3}}
$$

where $d$ is the thickness of the adsorbed film and $\alpha$ denotes the van der Waals constant $[5,6]$. The short range interactions usually correspond to molecular and atomic forces, such as electron exchange or hydrogen bonding. 
A widely used paradigm for the characterization of the wettability of a solid surface by a liquid [7] is the contact angle, 0 , which forms at equilibrium between the liquid/gas interface close to the solid surface, and the surface itself [1] (cf. Fig. 1a). For a perfectly homogeneous surface, it is uniquely determined by the interfacial tensions between substrate and gas, substrate and fluid, and fluid and gas, respectively. However, it tums out experimentally that small inhomogeneities of the substrate are sufficient to induce a large hysteresis in $\theta$ for the receding or advancing contact line $[8,9]$. Consequenty: the contact angle is, in general, a dynamical quantity which depends upon the history of the measurement, and its equilibrium value is not easily accessible.

It has proven more advantageous, both conceptually and experimentally, to consider a situation in which all involved interfaces are parallel to each other, i.e., to consider the thickness of a layer of adsorbate which forms at equilibrium on the substrate due to attractive forces (such as the van der Waals force) it exerts onto the (gas) molecules in the adjacent half space. The wetting properties of the solid surface with respect to the liquid are completely characterized by the functional depentence of the layer thickness upon the pressure in the gas phase.

For illustration, let us consider a closed container at a temperature $T$. filled with gas whose pressure can be adjusted up to satutated vapor pressure, $p_{\text {sar }}$. The chemical potential (of the molecules in the container), which we consider with respect to coexistence, is given by

$$
\Delta \mu=\mu-\mu_{c o s}=k T \ln \frac{p}{p_{s a t}} .
$$

Condensation will occur when saturated vapor pressure is reached, i.e, when $\Delta \mu=0$. It depends upon the wetting properties of the container walls whether condensation will proceed as the formation of a thick film at these walls (if $\theta=0$ ), or by nucleation of single condensate droplets (or crystallites, if the adsorbate is solid) at coexistence $(0>0)$, which form somewhere at the bottom of the container.

The corresponding dependence of the adsorbed film thickness (as a function of pressure) is depicted schematically in Fig. Ib. When the solid is wet by the adsorbed liquid (solid line), a homogeneous film forms on the substrate whose thickness diverges continuously as coexistence is approached. This situation, which corresponds to $\theta=0$ in the contact angle picture, is called "complete wetting". When the film is stabilized by the van der Waals interaction (cf. Fq. (1)) with the substrate $|6|$, it is easily seen by combining Eqs. (1) and (2) that the film thickness should diverge as

$$
d \propto(p a i t-p)^{-1 / 3}
$$

when saturation is approached. On the contrary, it may occur that the film thickness remains finite close to coexistence (dashed line). For this case, the term incomplete wetting' is used. Since a linite thickness $d$ is assumed by the film at coexistence, we may conclude that, in the contact angle picture, the droplet(s) on the surlace (which represent the condensate at liquid/vapor coexistence [10]) coexist with a film of thickness $d_{l}$. on the remaining parts of the surface.

These phenomena can be understood on a more fundamental level by considering the so-called effective interface potential, $\Omega$, which is defined as the excess free energy requited to bring the substrate/film interface and the film/gas interface together 
$\Omega$
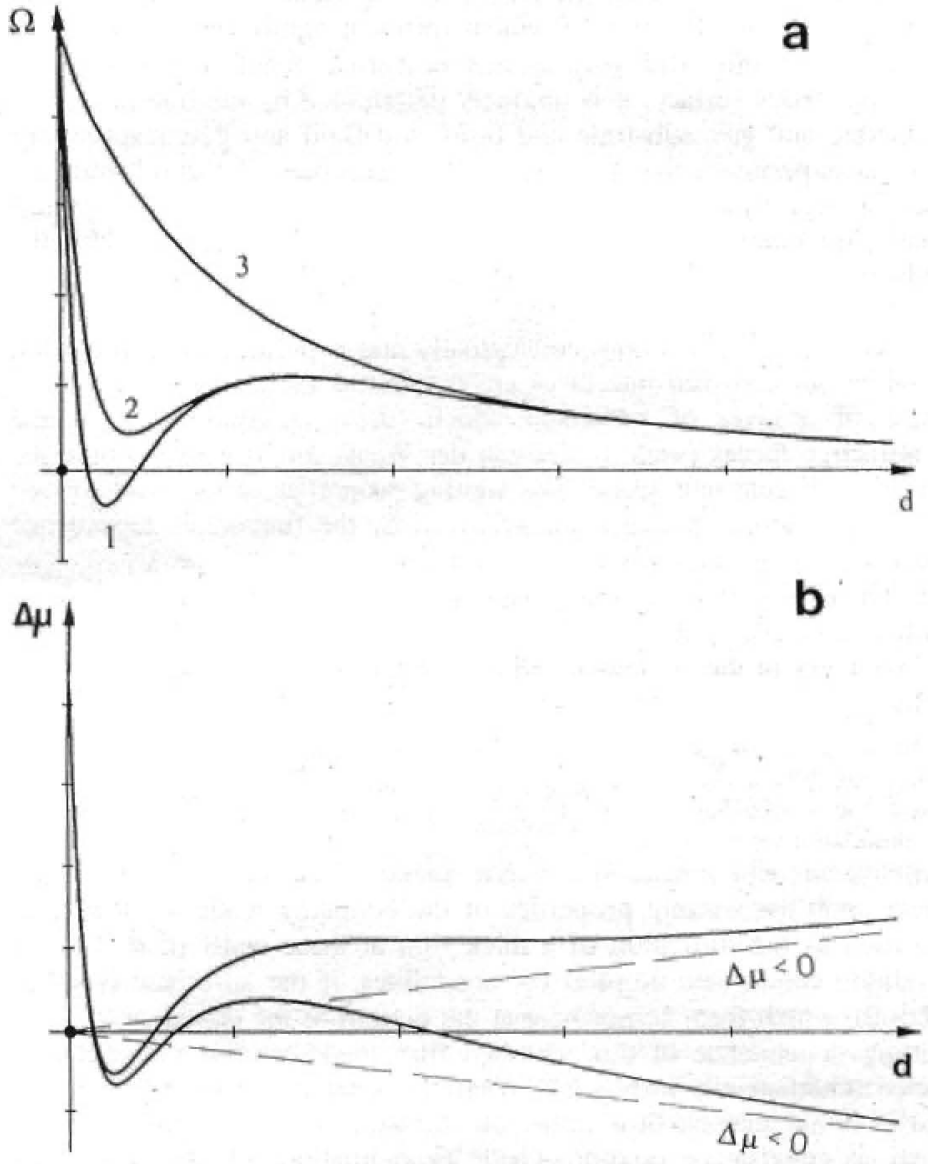

Fig. 2 a) The three types of effective interface potentials, $\Omega_{f}$, at coexistence, as discussed in the text. b) The situation off coexistence. An additional free energy, $-d \Delta \mu$, is required to form a film of thickness $d$. The global minimum determines the equilibrium film thickness.

from infinity to the distance $d$, i.e., the film thickness $[2,3]$. While the contact angle reflects integral properties of this potential, a measurement of the adsorbed film thickness as a function of gas pressure (adsorption isotherm) provides insight into its details.

As an example, let us consider an effective interface potential, $\Omega$, which consists of a long range van der Waals tail and some additional structure at small $d$ due to short range forces, as depicted in Fig. $2 \mathrm{a}^{2}{ }^{2}$ At coexistence, there is no cost, nor gain,

${ }^{2}$ It should be mentioned that although the van der Waals interaction between two single molecules is always attractive, the van der Waals tail of $\Omega$ may be either positive (as in the figure) or negative; roughly speaking, it is negative when the polarizability of the adsorbate is larger than that of the substrate [6]. 
in free energy for creating adsorbed (condensed) film material, thus, $\frac{\mathrm{d} \Omega(d)}{\mathrm{d} d} \rightarrow 0$ for $d \rightarrow \infty$, and the convention $\Omega(\infty)=0$ is chosen. The global minimum of $\Omega_{1}$ is at a finite thickness of atomic or molecular dimension, due to the short range character of the forces giving rise to the structure at small $d$. In this case, a thin film of thickness $d_{c}$ is stable on the surface at coexistence, which corresponds to incomplete wetting. On the contrary, if the minimum is not deep enough $\left(\Omega_{2}\right)$ or even absent $\left(\Omega_{3}\right)$, the global minimum (at coexistence) is at infinite thickness, corresponding to complete wetting.

Off coexistence, there is obviously an additional term to the free energy given by $-d \Delta \mu$, which denotes the penalty to be paid for creating the amount of condensate corresponding to the film thickness, $d$. The resulting behaviour of the total free energy of the film is depicted schematically in Fig. $2 \mathrm{~b}$ for $\Omega=\Omega_{1}$. It is immediately clear from the figure that there is a jump in tilm thickness from $d_{v}$ to infinity when one is crossing coexistence $(\Delta \mu-0)$, as shown in Fig. $1 \mathrm{~b}$ as the dashed line. It is also clear that for $\Omega_{2}$ and $\Omega_{3}$, there will be (at least asymptotically) a smooth divergence of the equilibrium film thickness (i.e., the global minimum of $\Omega_{j}-d \Lambda \mu$ ) upon approaching coexistence, as represented by the solid line in Fig. Ib. It is furthermorc clear that if we replace $\Omega_{1}$ by $\Omega_{2}$ in Fig. 2b, there will be a certain value of $\Delta \mu<0$ at which two minima coexist which are equally deep, corresponding to the coexistence of a thin film with a film of large, but finite, thickness. At this value of $\Delta \mu$, a finite jump in film thickness occurs upon approaching coexistence, which leads from a thin film to a thicker film whose thickness smoothly diverges when coexistence is approached further. This phenomenon is called prewetting.

Let us now consider a phase transition between complete and incomplete wetting. It may be imagined, for instance, that the strong variations in $\Omega_{1}$ at small $d$ will be smeared out thermally upon heating, leading to a potential of type $\Omega_{2}$. At a certain temperature $T_{t,}$, which is called the wetting temperature, there will thus be a transition from type 1 to type 2 in $\Omega$. Below that temperature there will be incomplete wetting, above there will be complete wetting. When the temperature is varied at coexistence, the adsorbed film thickness will jump discontinuously at $T_{m}^{\prime}$ as it is depicted schematically in Fig. 3 (dashed line). This behaviour is called a first order wetting transition, as it is observed with liquid helium on cesium surfaces. Since $\Omega$ crosses over from type 1 to type 2 , it is clear that a first order welting transition is accompanied by prewetting. This will be discussed in more delail below, in the context of the wetting properties of liquid helium. The conditions for a continuous wetting transition, as represented by the full line in Fig. 3, are more subtle, and a

Fig. 3 Schematic representation of a wetting transition along a coexistence curve which occurs at the wetting temperature, $T_{W}$. Solid line: continuous transition.

Dashed line: 'first order' wetting transition.

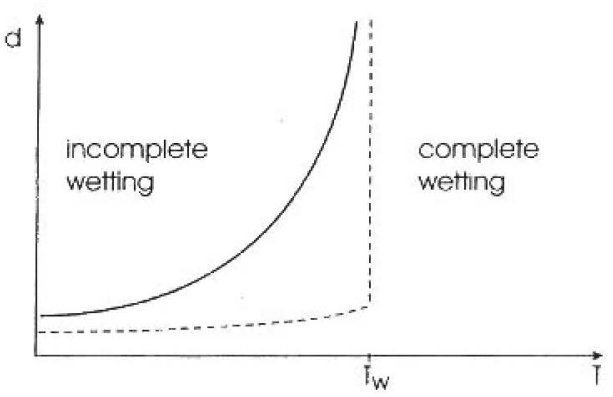


discussion would go beyond the scope of this paper [11]. It should be noted, however, that it has recently been observed for the first time with pentane adsorbed on water, somewhat above room temperature [12].

We have repeatedly used the notion of an infinitely thick film. It is clear, however, that, in a real experiment, there will never be a film thickness which is even close to infinite: as soon as the film thickness is large enough for gravitational elfects to become comparable to the van der Waals altuaction by the walls, the condensate will gather at the botum of the container instcad of forming a thick film at the wall. Furthermore, even slight temperature gradients or fluctuations tend to result in thinning the film [13]. The typical signature of a welling Iransition is a change in film thickness from a few monolayers (incomplete wetting) to at most several hundred Angstroms (complete wetting).

Aside from the wealth of interesting phenomena at cquilibrium, there is a huge variety of effects when non-equilibrium situations are concerned. A typical example is the dynamics of dewetting, to which a large number of recent publications has been dedicated [14-17]. Since there are numerous interactions and transport mechanisms involved in both the statics and the dynamics of wetting and dewetting, and their interplay can give rise to a wide variety of phenoment, there is a strong need for simple model systems which enable the study of basic mechanisms in a clean and controllable way. Well known examples of such model systems, which have been used in a large number of studies of other phenomena, are polymers (for the sake of controllability) and quantum liquids, like superfluid helium, because of their well defined and well known properties [18].

In the present paper, we want to concentrate on the quantum systems helium $\left({ }^{4} \mathrm{He}\right)$ and (molecular) hydrogen as model adsorbates. The mutual interactions of hydrogen molecules and helium atoms are known more accurately than for any other system. Furthermore, the possibility of cooling into a superfluid state in the case of helium yields an additional degree of freedom which allows, among others, 10 study the influence of viscosity in the dynamics of wetting phenomena [19-21]. Finally, it must be noted that the necessity of cooling to low temperatures effects in freezing out almost all impurities [22]. This is a great advantage since wetting phenomena are particularly sensitive to impurities, as mentioned above, which are always present at room temperature.

As far as wetting phase transitions are concerned, the choice of hydrogen and helium suggests itself, since hydrogen exhibits a continuous wetting transition (at the triple point) [23] on all substrates studied to date, and helium undergoes a first order wetting transition on cesium surfaces [24-26]. It is these Iwo phase transitions on which the present paper concentrates. Reviewing some of our recent work, we present optical investigations which elucidate both the statics and dynamics of the systems close to the transitions.

\section{The probe: optically excited surface plasmons}

Surtace plasmons (SP) are electromagnetic waves travelling on metal surfaces. 'Their typicat frequencies are in the visible range of the spectrum which makes them well suited for use as an optical probe of processes taking place in the close vicinity of the surface [27], such as wetting phenomena. Light can be coupled to the SP by means of an evanescent wave, as for instance by illuminating the base of a prism un- 


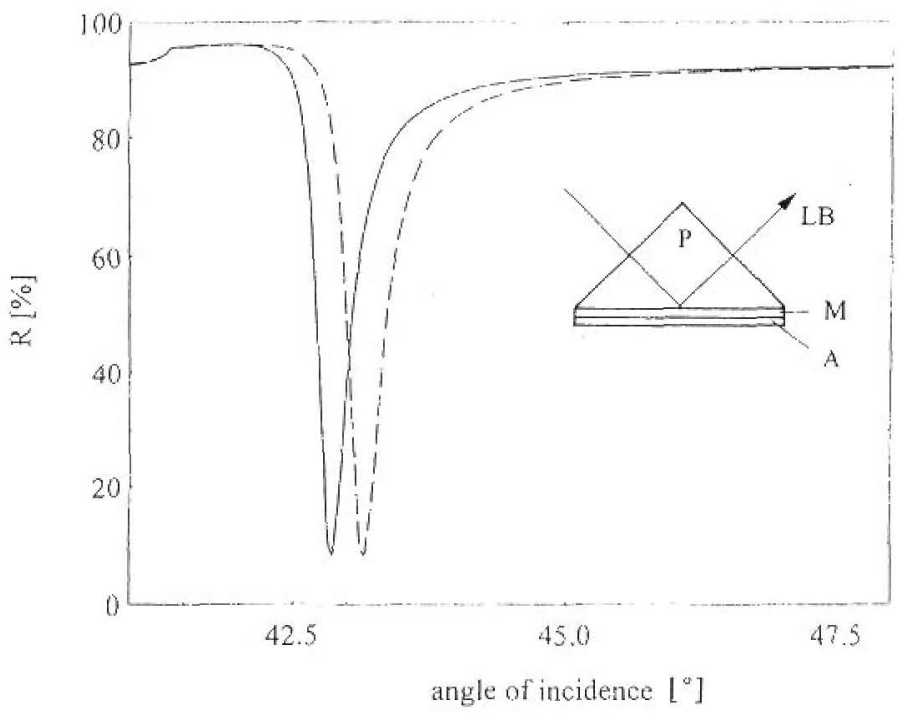

Fig. 4 Reflected intensity vs. amgin of incidenee in a surface plasmon spectroseopy experiment. Inset: sketch of the experinental selup, showing the prism (P), the laser beam (LB), the metal film (M) supporting the surface plasmons, and the adsorbate (A).

der total internal reflection conditions (inset in Fig. 4). When the metal whose surface is to be investigated is evaporated as a film of appropriate thickness (several hundred Ångstroms) onto the prism base, SP on the metal/vacuun interface are resonantly excited at a particular angle of incidence, $O_{0}$, at expense of the reflected light. This results in a pronounced minimum in the reflected intensily, as shown in Fig. 4 for a $500 \AA$ silver film (solid curve). The presence of a thin film of, say, hydrogen on the metal surface detunes the SP resonance (dashed curve). This allows such a film to be detected with an accuracy of better than an Angstrom thickness if a noble metal is used, and if the resonance angle is measured with sufficiently high resolution [28]. It should be noted that the measured film thickness is in fact the coverage, averaged over a mesoscopic distance given by the decay length of the surface platsmons. The latter is typically several microns, depending on the metal used $[29,30]$.

In general, it can be judged that the disadvantage of this method lies in the restriction to metal films as the substrates (there are methods to circumvent this restriction, but they require some preparational efforts [31]). Its advantage is to be seen in its simplicity, its small sensitivity to polatization effects, such as strain-induced biretrimgence in the cryostat windows, and its capability of last dynamical recordings. 


\section{Triple point wetting of hydrogen: a continuous wetting transition}

\subsection{Wetting properties close to the triple point}

It has been known for a long time that most adsorbates, and hydrogen in particular, do not wet solid substrates below the melting temperature [23, 32-37], even if the substrate is wet by the liquid phase. In other words: solid films on solid substrates are usually not thermodynamically stable, a fact which is of great significance in modern thin film technology. As a consequence, there is a transition between dry and wet at the melting temperature of the adsorbate, a behaviour for which the term triple point wetting has been coined. An explanation which is frequently given is that, in the solid phase, there is very likely a mismatch in the lattice constant of the adsorbate and the substrate material which causes additional strain in the adsorbed layer. This makes it unfavorable for the solid film to be formed [32]. However, the striking universality of triple point wetting (of nonwetting of the solid phase), which is observed even in systems in which the lattice constants of the solid adsorbate and the substrate are very close to each other [38], remains puzzling. In particular, triple point wetting occurs also on poorly defined substrates [39], which makes it hard to believe that the lattice mismatch is really the dominating effect.

As an example, let us now discuss experimental studies of the wetting properties of hydrogen in the vicinity of the triple point temperature, $T_{3}$, in order to compare with what one would expect from the above considerations. Fig. 5a shows the equilibrium film thickness of a hydrogen film adsorbed on a gold surface, as measured with optically excited surface plasmons [39]. The temperature is being varied along coexistence. Above the melting temperature, there is a large, but finite, thickness which is independent of temperature and corresponds to complete wetting. Below the melting temperature, two regimes can be distinguished. In an narrow range below $T_{3}$, the film thickness jumps hysteretically as one would expect for a first order wetting transition (it will be shown below, however, that this hysteresis is not inherent to the wetting transition itself). For lower temperatures, there is a gradual decrease in film thickness, which is shown in Fig. $5 \mathrm{~b}$ on a $\log / \log$ scale, as a function of reduced temperature, $t:=\left(T_{3}-T\right) / T_{3}$. As one can see, the data seale algebraically, with an exponent of $-1 / 3$ (dashed line). This behaviour is reminiscent of a continuous phase transition.

In order to discuss the observed behaviour, we consider the phase diagram of hydrogen as depicted schematically in Fig. 6. The liquid/gas coexistence line, which represents the intersection of the free energy surfaces of the liquid and the gaseous phase, is extended by the dashed line into the solid phase region. We know from our data above $T_{3}$ that the film thickness becomes 'infinite' when this coexistence is approached (according to Eq. (3)), indicating complete wetting. On approaching the triple point along the solid/gas coexistence line which intersects the liquid/gas coexistence line at a finite angle, one would thus expect the adsorbed film thickness to diverge as $t^{-1 / 3}$, consistent with what is observed at lower temperatures below the triple point (Fig. 5b). We are led to the interpretation that the continuous increase of the film thickness upon approaching the triple point from below along solid/gas coexistence is physically identical to complete wetting at liquid/gas coexistence.

If this is the correct interpretation, one would expect that adsorption isotherms (dotted line in Fig. 6), taken at temperatures below the melting temperature, behave as if they would diverge at the extrapolated liquid/vapor coexistence within the solid phase region (dashed line in Fig. 6). Fig. 7a shows a number of isotherms taken be- 
Fig. 5 Hydrogen film thickness adsorbed on at gold surface at coexistence, as a function of temperature. a) Linear scale: as it turns out, the hysteresis is not an indication of a first ordes Iransition (see text), b) Log/ log scale: vs. reduced temperature, $\left(T_{3}-T\right) / T_{3}$. The slope of the dashed line is $1 / 3$.
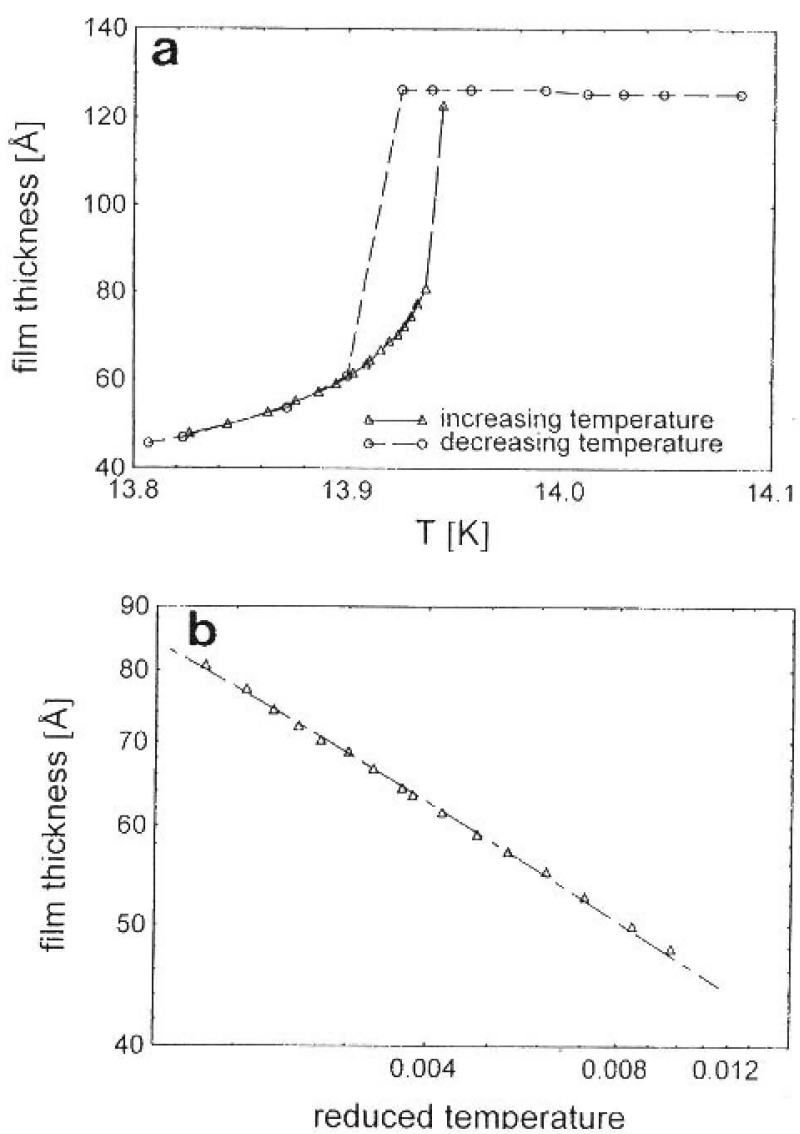

low the triple point. For cross checking with our results above, we show in Fig. 7b their intersection points at coexistence $\left(d_{c}\right)$ in a $\log / \log$ plot as a function of reduced temperature. Obviously, the scaling law discussed above for experiments along the sublimation curve is reobtained. All of the isotherms displayed in Fig. 7 a are found to be well represented by vin der Waals isotherms according to Eq. (3) when $p_{s u r}$ is replaced by a free parameter, $p_{0}$. II' we take the poles of the fitted functions (at $p=p_{0}$ ) and plot their corresponding pressures as a function of temperature, we obtain the plot shown in Jig. 8, where the poles are depicted together with the solid/vipor coexistence curve. Within experimental scattering, the poles are consistent with the extrapolated liquid/vapor coexistence curve represented by the dashed line.

An important implication from the above findings is that the hydrogen film close to (but below) the triple point is liquid, since complete wetting at liquid/vapor coexistence takes place via liquid film adsorption. Due to the small amount of material which these films provide and the small scattering cross section of hydrogen, it would be difficult to determine the morphology of the film material directly by $\mathrm{x}$-ray 


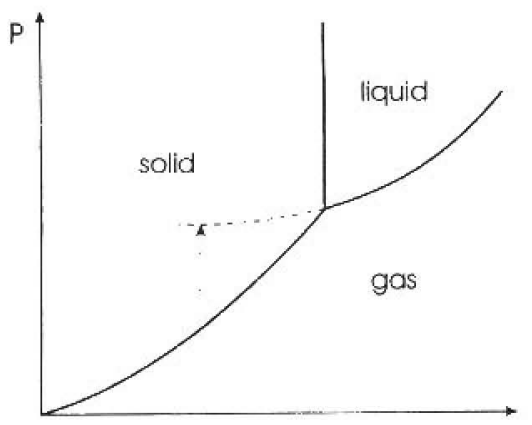

Fig. 6 Schematic phase diagram of hydrogen. The liquid/gas coexistence curve is extrapolated into the solid phase region (dashed line). The dotted line indicates the path of an adsorption isotherm, extrapo-

$T$ lated up to liquid/gas coexistence.
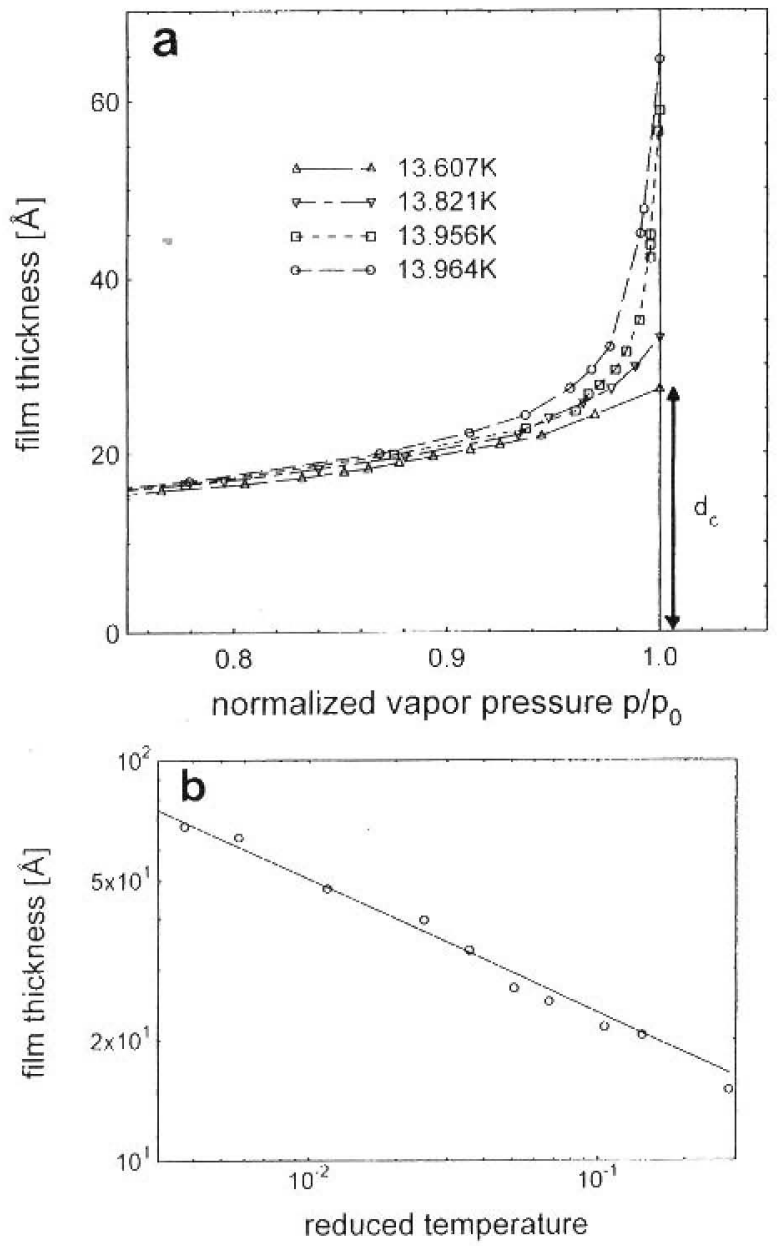

litg. 7 a) Adsorption iso. therms for hydrogen on gold at different temperatures. The film thickness which remains finite al coexistence is denoted by $d_{i}$, b) Log/log plol of the intersections of the isotherms with coexistence $\left(d_{\ell}\right)$, corresponding to the datit shown in a). The slope of the solid line is $-1 / 3$. 
Fig. 8 Locus of the poles, $p_{0}$ of van der Waals isotherms fitted to the data shown in Fig. 7a, plotted in the $p-T$ plane. The solid line represents the sublimation curve, the dashed line represents the fextrapolated) liquidigas coexistence.

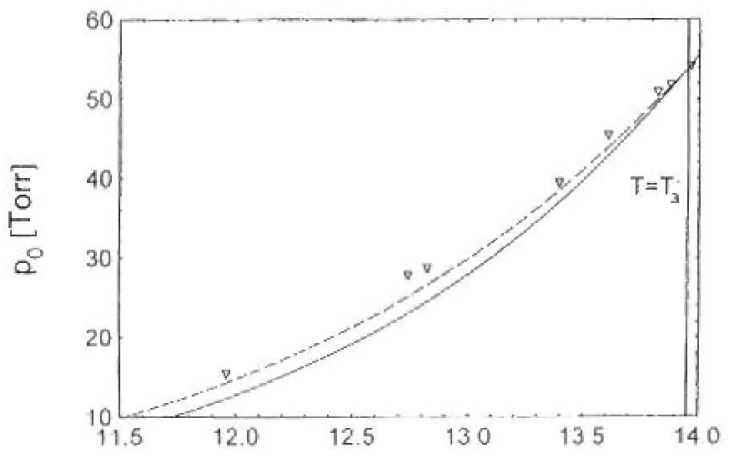

$T[\mathrm{~K}]$

scattering techniques. However, one of us (R.C.) has recently performed $x$-ray experiments on a similar system which exhibits triple point wetting as well, namely $\mathrm{Ar}$ on $\mathrm{MgO}$ [40]. The larget scattering cross section of argon allowed the determination of the film morphology, and it turned out that there is only fow solid material in the film. Close to the triple point, most of the film is actually liquid.

In fact, it has been shown theoretically that the mere compression of the film by the van der Waals pressure gives rise to a substantial increase in the free energy [41]. The key idea is that since the film is adsorbed on a solid substrate, its lateral extension is fixed, and the van der Waals pressure can compress it normally to the surface, but not laterally. As a consequence, there is a shear in the solid film in addition to the compression, while the liquid film experiences only the compression (the shear modulus of at liquid is zero!). That a shear considerably increases the free energy of a system can be seen directly from the Grinfeld instability $[42,43]$, which shows that a solid under shear may try to minimize this shear even by changing its overall shape. In our case, it gives rise to an additional, negative term in the effective interface potential, $\Omega$, which results in a global minimum at finite thickness: the surface is not completely wet. The most promising feature of this model is that it does not rely on the atomic or molecular structure of the constituents, but considers only continuum quantities, like the Poisson constant and shear modulus of the adsorbate. It is thus capable of explaining the observed ubiquity of triple point wetting. In conclusion, we are led to believing that even below the triple point, only a very thin film of solid hydrogen (approximately 3 layers) is thermodynamically stable. The formation of a thick film as one approaches $T_{3}$ proceeds via adsorption of liquid material by virtue of complete wetting at liquid/vapor coexistence. The 'triple-point-wetting' transition is thus to be viewed as physically identical to a continuous wetting transition.

\subsection{Supercooling effects near the triple point}

It had been mentioned above that aside from the theoretically expected hehaviour, there was a hysteresis observed reproducibly also in former surface plasmon experiments, which occurred within about $100 \mathrm{mK}$ of, and below, the melting temperature 

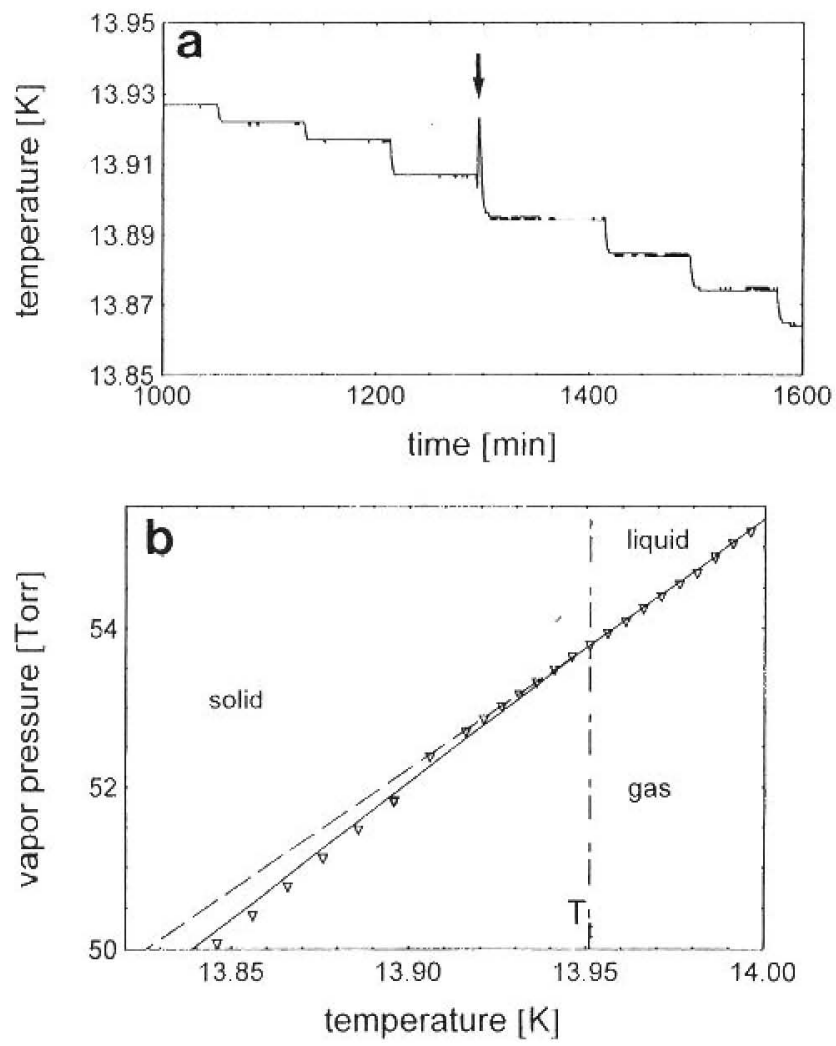

Fig. 9 a) Cell temperature during slepwise cooling, slightly below the melting temperature. At onc of the temperature steps, there is a significant peak which is indicated by the arrow. This is alue to the latent heat liberated upon solidification of the hydrogen in the cell. b) Cell pressure vs. temperature, measured during the same run as a). The data follow the liquid/vapor coexistence curve antil well below the triple point, the pressure jumps to the value corresponding to solid/vapor coexistence. Solid lines: phase coexistence curves. Dashed line: liquid vapor coexistence extrapolated into the solid phase region, as in Fig. 6.

[44]. This seems to be at vatiance with the other findings discussed above which point to a continuous phase transition. However, if one records the pressure and temperature in the coll accurately enough, one can see that this hysteresis is in fact due to supercooling of the bulk phase, which is in equilibrium with the film. Fig. 9a shows a chart recorder trace of the cell temperature. The temperature is reduced stepwise, and ample time is left for equilibration after each change. At one particular step (arrow), there is a large peak in temperature which we attribute to the latent heat liberated when the bulk hydrogen in the cell solidifies. The cell pressure is plotted as a function of the cell temperature in Fig. 9b. It is clearly seen that it follows the liquid-vapor coexistence curve (dashed line) until well below the triple point. Upon 
solidification of the bulk phase, it jumps to the solid-vapor cocxistence curve. This jump occurs at the same time as the temperature peak shown in Fig. 9a.

\subsection{Wetting properties at lower temperatures}

According to the above, at temperatures far below the triple point, only very thin films should be stable on the substrate. This has important implications as, e.g., for nuclear fusion experiments $[45,46]$ and experiments on the neutrino rest mass [4749 , where the preparation of thick solid hydrogen films is of crucial importance. By quench condensation onto sufficiently cold substrates, it is of course possible to prepare films of solid hydrogen which are much thicker than the equilibrium film thickness expected for a solid film. This is due to the fact that, at low temperatures, the molecular mobility along the surface is so low that hydrogen molecules reaching the surface will not be able to arrange into an energetically more favorable configuration. Instead, they will remain more or less at the place where they hit the surface, resulting in a uniform film. However, this is a nonequilibrium state of the system, which will be very sensitive to heating. Even small thermal leaks may suffice for lateral transport to become thermally activated. The system will then attain an energetically favourable configuration, such as an ensemble of droplets in coexistence with a thin film $[50,51]$.

Such a rearrangement is optically detectable, since the formation of an ensemble of droplets causes turbidity, which is visible, e.g., in the surface plasmon signal. Fig. 10 shows both the surface plasmon resonance position and the resonance width

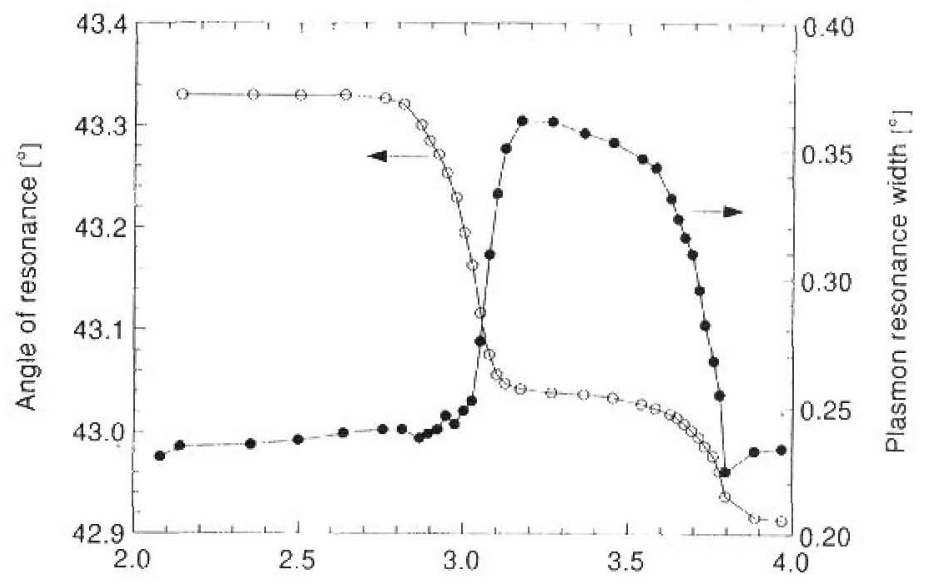

Temperature $[\mathrm{K}]$

Fig. 10 An annealing experiment with hydrogen on a gold surface. Shown is the surface plasmon resonance position (left scale, open circles) and the resonance width (right scale, full circles). Before the material evaporates above $3.5 \mathrm{~K}$, there is a clear transition to a morphologically modified state at about $3 \mathrm{~K}$. This is interpreted as due to the formation of small droplets, which coexist with a molecularly thin film. 


\section{$\mathrm{H}_{2} / \mathrm{Ne} / \mathrm{Ag}$}

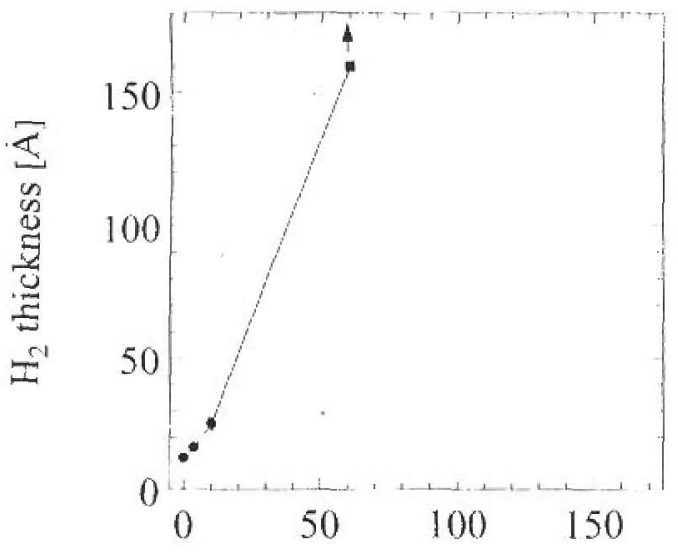

Ne-thickness $[\AA]$
Fig. 11 Wenting of a compusite $\mathrm{H}_{2} / \mathrm{Ne}$ film on a silver surface. The hydrogen film thickness which can be prepared at equilibriun as a homogeneous film is plolled vs. the neon-preplating thickness. Al a neon thickness of $60 \AA$, complete wetting is observed.

during a typical annealing experiment with a quench condensed hydrogen film. The film had been prepared at $1.5 \mathrm{~K}$, where no appreciable lateral transport takes place and the film is uniform. Upon heating to about $3 \mathrm{~K}$, lateral transport sets in and the adsorbed material rearranges into an ensemble of droplets. The redistribution of material at the interface changes the effective polarizability "seen" by the surface plasmons, resulting in the observed shift of the resonance anglc. At the same time, the droplets give rise to diffuse scattering which increases the surface plasmon damping and thus the width of the resonance [50]. Only upon heating to above $3.5 \mathrm{~K}$, the hydrogen evaporates.

An alternative way to pinpoint the dewetting effect is to deposit the hydrogen at temperatures high enough for the film to remain at thermal equilibrium during the deposition process. One then observes first a uniform deposition up to the equilibrium thickness, and then, at a certain coverage, a drastic increase in the intensity of the light scattered diffusively into the adjacent half space $[50,52]$. This indicates the growth of droplets upon the homogeneous film. The occurrence of droplets during film growth at equilibrium is another fingerprint of incomplete wetting at low temperatures, since this indicates a finite contact angle. The thickness at which droplet formation sets in is again the equilibrium thickness $d_{c}$ discussed in the introductory section.

This kind of 'toughening' due to incomplete wetting is very disturbing for' experiments which need unifom solid films of larger thickness, as those mentioned above. Can we apply our knowledge of wetting mechanisms to tune the wettability of the substrate so as to circumvent dewetting upon annealing? We have discussed above that the van der Waals pressure close to the substrate induces a shear in the solid film which gives rise to dewetting below the triple point. If we were able to reduce the van der Waals attraction of the substrate in a controlled manner, it might be possible 
to find a certain lange of parameters where complete wetting occurs. One way to reduce the van der Waals attraction of the substrate is 10 coat it with a material whose van der Waals attraction is smaller than that of the substrate material. The question is whether this composite substrate may now be completely wet by hydrogen.

We have tested this possibility by preplating our metal substrate with a well controlled amount of neon, before hydrogen was deposited. We then measured the amount of hydrogen which could be deposited on the neon-coated substrate without the formation of droplets, as revealed optically. This maximum thickness of a homo* geneous hydrogen film which was stable at equilibrium conditions is plotted in Fig. 11 as a function of the neon preplating thickness. It is clearly seen that, as expected, the equilibrium thickness increases with increasing neon film thickness. At a neon thickness of 60 Angstroms, we could still not see any indication of turbidity at the largest hydrogen film thicknesses we could prepare, which we interpret as an indication of complete wetting.

\section{Liquid helium on a cesium surface: a first order wetting transition}

After having explored triple point wetting and its physical reasons by means of hydrogen films as a model system, let us now turn to liquid helium. As opposed to the continuous transition we have discussed with hydrogen, it provides a first order wetting transition on cesium surfaces, with the particularity of being superfluid below a certain temperature. By improving our surface plasmon resonance setup to yield a surface plasmon mictoscope, we will concentrate on morphological and dynamical aspects of liquid helium droplets impinging on a slanted cesium surface.

\section{I The phase diagram}

One of the most striking features of liquid helium is that it creeps out of any container into which it has been poured. This is due to two remarkable properties: first, helium wets the container wall completely since, owing to its exceptionally small polarizability, its van der Waals interaction with the wall is always stronger than with itself. This gives rise to an effective interface potential which favours a thick liquid film on the wall. Secondly, this film is capable of supporting macroscopic material transport due to superfluidity (below $T_{2}=2.17 \mathrm{~K}$ ). As a result, the liquid will leave the container by virtue of the film, which covers both the outer and inner wall sur. lace. This property can be quite annoying in cryostat design or in any low temperature experiment.

It was therefore quite a stir in the community when non-wetting of helium on some alkali metals below a certain temperature was predicted theoretically [53]. The idea was that although the van der Waals term in the effective interface potential favours wetting, there is, at small distance from the wall, a strong repulsion due to the very large Bohr radius of the outer shell clectrons of the alkali metal atoms. This is strong enough to render $\Omega$ of type 1 (cf. Fig. 2a), which corresponds to incomplete wetting. An intense experimental activity emerged, which soon led to the confirmation $[24,26]$ of a wetting transition of liquid ${ }^{4} \mathrm{He}$ on a cesium surface. The wetting temperature was found to be around $2 \mathrm{~K}$, with some dependence on the substrate quality. Thorough studies using the quartz microbalance technique have fully estab- 


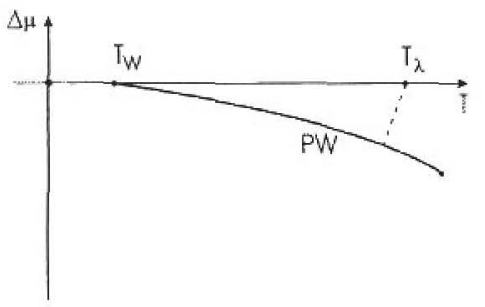

Fig. 12 Schematic wetling phase diagram of liquid ${ }^{4} \mathrm{He}$ on cesium. Liquid/gas coexistence conesponds 10 $\Delta \mu=0 . T_{W}$ and $P W$ denote the weting temperature and the prewetting line, respectively. The superfluid transition is indicated by $T_{\partial}$ and the Kosterlitz-Thouless line (dashed).

lished the nature of the wetting transition on cesium as first order wetting $[25,54$, 55], and it seems that there is no finite wetting temperature on the other alkalli metals.

Fig. 12 shows the wetting phase diagram of liquid ${ }^{4} \mathrm{He}$ on cesium schematically. At a certain temperature, $T_{W}$, the effective interlace potential crosses over from type 1 to type 2, corresponding to a discontinuous transition from incomplete wetting below to complete wetting above $T_{W}$. The phenomenon of prewetting, which accompanies a first order wetting transition as discussed in the introduction, manifests itself in a prewetting line ( $\mathrm{PW}$ ) which denotes the coexistence of a thin and a thick film. It ends at a critical end point, at which the difference in film thickness between the coexisting films vanishes (this corresponds to the disappearance of the inflection points in the effective interface potential, $\Omega$ ). It extends to temperatures above the lambda temperature, such that there is an intersection between the prewelting line and the Kosterlitz-Thouless line (dashed) which describes the superfluid/normal transition in the liquid film. Details like the slope of the prewetting line are still not completely setled [56], but the qualitative shape of the phase diagram is well established.

\subsection{Droplets impinging on a slanted surface}

In order to image the impact and flow of droplets of liquid helium on a cesium surlace, we performed in-situ surface plasmon (SP) microscopy. A SP microscope results when a sample like the one shown in the inset Fig. 4 is illuminated with an expanded laser beam and the reflected light is imaged onto a CCD camera chip 129 , 30]. Since the sensitivity of the method rests on the weak damping of the surface plasmons, the decay length of the latter may be as large as 20 microns (as in the case of silver), which is then the limit of spatial resolution. If other metals are used, the sensitivity is somewhat inferior due to higher surface plismon damping but, at the same time, the lateral resolution is better. Although the film thickness resolution is not as good as in a high resolution optical microscope, helium films with a few Angstroms thickness can be imaged with reasonable contrast and a temporal resolution limited only by the recording equipment. The most striking difference to the setup used for the experiments with hydrogen discussed above is that the angle of incidence is kept constant here, while the information of the adsorbed thickness is encoded in the intensity of the reflected light.

In our experiment, standard video equipment was used to record the dynamics of superfluid ${ }^{4} \mathrm{He}$ droplets falling onto a tilted, cesiated surface. in Fig. 13a, we depicted our sample cell which contains the prism (P), a capillary above the prism for supplying the liquid helium and a $C$ s dispenser (D). Before the prism was mounted in the 


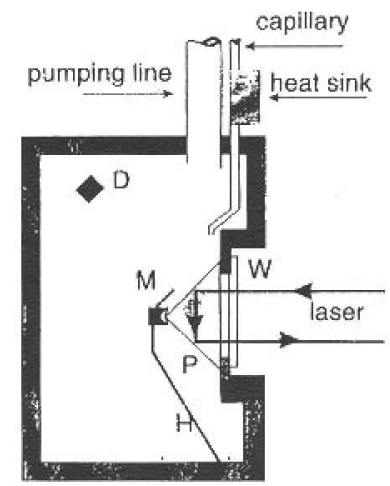

Jig. 13 a) The sample cell used for optical experiments with liquid helium fihus on cesum surfaces. During the experimen, the cell is completely inmersed in a liepuid helium? bath. The optical path traverses the helium and nitrogen batlss of the glass cryostat assembly. The mak (M) serves for blotkng cesium deposition trom the dispenser (D) antu the lowe part of the prism fited b) SP intige of the upper pristn fice. The upper, darker part is covered with $\approx 10$ atonic linyers of cesium atop of a silver lillm, which remaned uncovered in the lower, brighter part hy virtue of the mask ( $\mathrm{M}$ in a). The difference in grey valthe is due to the fact that at the angle of incidence chosen. SP iare resolamtly excited only in the upper, cesiated pirt.
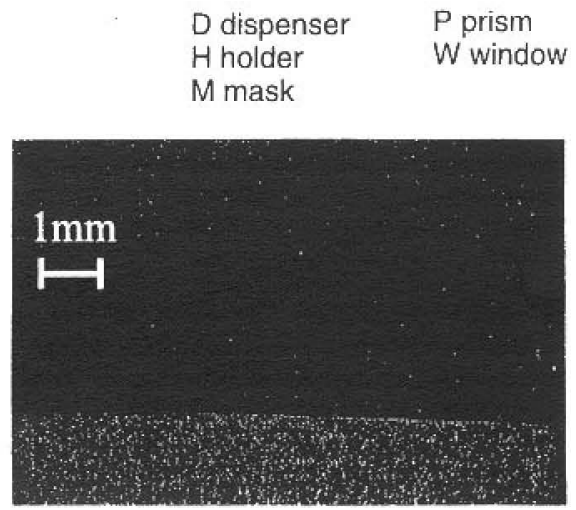

cell, its faces (cathetes) were covered with a $33 \mathrm{~nm}$ silver film. On the upper prism face, this served for reducing the surface plasmon damping: using an all cosium film would lead to a very large resonanee width and, correspondingly, a poor contrast. On the lower face, il provides a melal mirror necessary for imaging. After cooling to $4.2 \mathrm{~K}$, the dispenser is heated and approximately ten atomic layers of Cs are deposited onto the upper prism face. This has been shown to yick a welting tenperature around 2 K [25,57]. A mask (M) has been placed in from of the lower part of this prism face in order to provide a non-cesiated silver sulate for comparison. This can be seen in Fig. $13 b$ which shows a SP microscopic image of the upper prism tace, with the angle of incidence adjusted so as fo match the Sip resomance on the cesiated part. The latter thus appears dark. while the siver part, which is not exactly in resonance, appears brighter.

\subsubsection{Below the wetting temperature}

When a ${ }^{4}$ He droplet falls down onto the upper prism face, the SP in the region covered with heliun will be detuned resulting in a substantial increase in brighucss. This is shown in Fig. 14, which shows the surface 140 ms after a doplet hats arived 


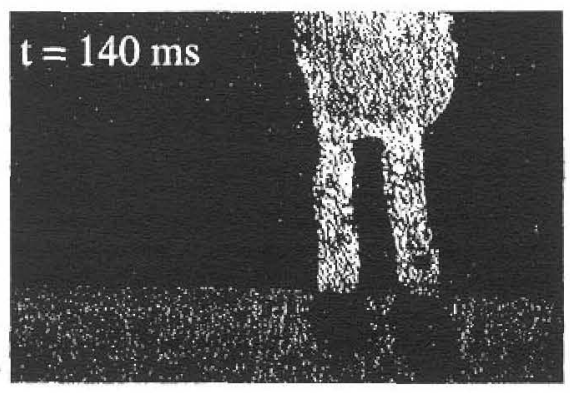

Fig. 14 SP microscopy of a hicpuid hedium droplet, $140 \mathrm{~ms}$ after impatet on the cesiated sur'face. lasteat of spreating on the surfice, the liquid forms lwo chanel-like structures though which it drains clown. As at rameles the silverul part, vigorous spreiding takes planct.

al contacl. Channel-Jike structures are clearly seen to evolve, indicating that the surface is not wet by the helium, the latter tending to develop a sharp contact line. It is observed that the helium flows down only along the finger-shaped channel regions generated intunediately after contact with the substrate. No tendency of spreading is apparent. However, as soon as the helium reaches the silver surface, the liquid llow, which had been restricted to 'channels' on the cesiated part of the simple, spreads laterally on the silver surface. The black regions in the silvered part indicatc a helium coverage which is enough to tune the SP on the silver into resonance. At the angle of incidence chosen, this is the case when the helium film is thick enough to appear as bulk helium to the evanescent wave of the SP. We can thus conclude that the black regions correspond to those parts on the silver which are covered by more that $750 \mathrm{~mm}$ of liquid helium. Since the thickness on the cesiated regions outside the channels is at most a few Angstroms, this demonstrates the strong spreading tendency of the helium on the silvered parts of the sample. In fact, carcful examination ol the grey values revealed a change in intensity virtuilly all over the silvered part.

It should be noted that a lull contrast (dark-bright) change on the cesiated part is acheved by coverage with approximately I $\mu$ m of helium. On the railing edges of the fingers wn the $\mathrm{Cs}$ (when the flow-down process of the drop was almost complete), we observed a quite gradual change in intensity over approximately one millimeter. indicating. that what is flowing are not bulk fluid structures, but are helium films. From the wetted area of several square millimeters and a typical droplet volume of a few hundred picoliters, we can estimate the film thickness to be a lew microns (cortesponding optically to bulk helium, as mentioned above). In this case, one expects a critical flow:velocity for the formation of quantum vortex rings on the order of a few $\mathrm{cm} / \mathrm{s}[18]$. In facl, analyzing a series of pictures, we found the flow velocity to be approximately $5 \mathrm{~cm} / \mathrm{s}$ which suggests that the film flow by which the drop drains down the surface is limited by the critical flow velocity.

When the liquid helium droplet has drained away, it is observed that a thick film remains which is stable for a time on the order of a second. Fig. 15 shows transient measurements of the film thickness during the impact and dramage of to consecutive droplets. In this case, the remaining 'metastable' film was less than $100 \AA$ thick, but also thicker films have been observed. The existence of metastable helium films on cesium is in fict well established $\lceil 54\rceil$ and may be well understood qualitatively. Since there is no material which is as dry by helium as cesium, defects on the cesium surface, whether these are holes in the cesiun film on dust particles, are always wet 


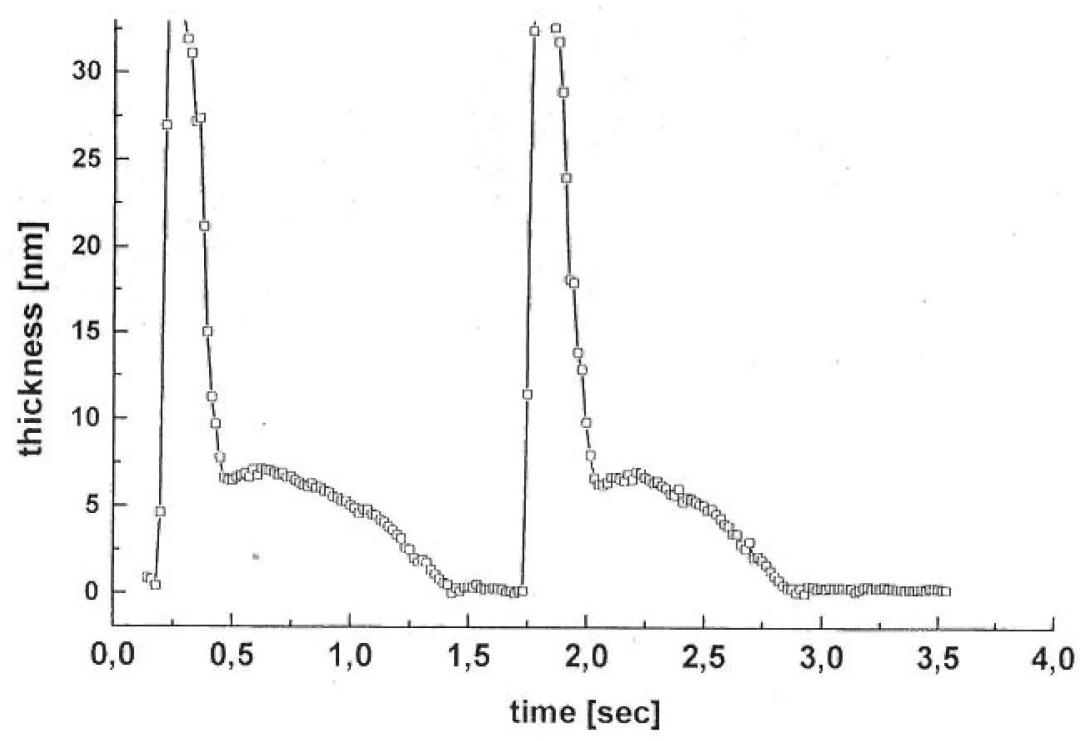

Fig. 15 Film thickness transients at a point of the simple which is repeatedly hit by a droplet. The melaslable film remaining for about a second after a droplet has drained away is clearly seen.

by helium. Contrary to what is usually experienced with other wetting layers, there is thus no nucleation center for dewetting. Furthermore, since the long range tail of the effective interface potential is in favour of wetting (due to the small polarizability of the helium atoms), there is no physical reason for spinodal dewetting to occur [16]. There is thus no simple way for the helium film to get off the cesium surface once it has been successfully deposited. When there is no dry (cesium) patch in the sample cell where dewetting can start, these metastable films may last for hours [54]. In our case, there are dry regions to nucleate dewetting outside the impact region of the droplet. However, as we could see in Fig. 14, the contact line is strongly pinned and must be moved in order for dewetting to proceed. Consequently, our somewhat preliminary interpretation of the dewetting dynamics of the metastable film displayed in Fig. 15 is that it is mainly dominated by the pinning forces which must be overcome to move the contact line over the cesium surface. The ultimate limitation of the lifetime of the film is given by its evaporation, since we are slightly ofl coexistence.

\subsubsection{Effect of the prewetting transition}

Before we discuss effects occurring at higher temperatures, let us first consider what it means thermodynamically when a droplet impinges on the cesium surface. Before the droplet touches the surface, there is already a thin film of helium which corresponds thermodynamically to the base pressure in the cell. In our experiment, this was very close to coexistence with the bulk phase $\left(p \approx 0.995 p p_{\text {sur }}\right)$. When the droplet touches the surface, the latter is in contact with the condensed phase, which corresponds to a state in the condensed phase region, or at least on the coexistence curve. We can thus envisage the impact of a liquid helium droplet as a small excursion in 
(a)

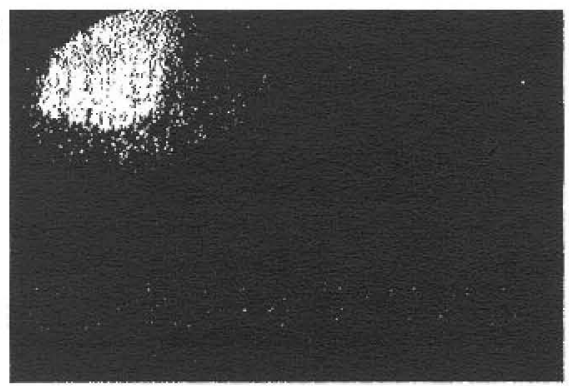

(b)

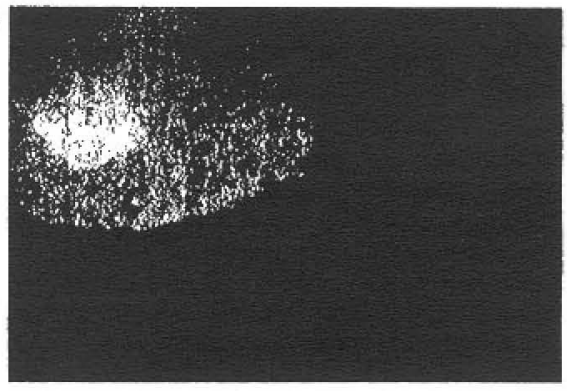

Fig. 16 Droplet morphology at sligghtly elevated comperatures, when the puewerting line is crossed themodynamically during droplet impres. "Ihe 'patnake?" intm ath he clealy discemed. The lime elapsed hetween the twi inages (a and b) is 201015 .

the phase diagram (cf. Fig. 12) which starts somewhat away from the coexistence curve $(A / t<0)$, approaches and meets with it, and finally returns to ils original posilion again when the droplet has drained away.

A glance at the phase diagram shows that such an excusion al temperatures slightly above the wetting temperature, $T_{w}$, should cross the prewetting line at some point. It is thus interesting to look for anomalies in the spreading behaviour of the liquid on the solid substrate which are to be attributed to crossing the prewelting line in the phase diagram. Note that it denotes the coexistence between a thin and a thick film. It should furthermore be emphasized that when the dioplet is on the surface already, the lateral distance from the droplet comesponds also to the themodynamical 'distance" from coexistence, $\Delta \mu$.

Fig. 16 shows the impact of a droplet at $1.85 \mathrm{~K}$, slightly above the wetting temperalure, which wats $1.80 \mathrm{~K}$ for this sample. There is now an internal structure in the droplet is it is deposited on the cesium substrite. In the center, the bright spot indicates the presence of 'bulk' helium, having completely detuned the surfice plasmon resonance. However, there is in addition an outer region with a rather uniform thickness, as can be judged from the unform grey value. It has a sharp boundary with the lest of the sample surlace. This surrounding film, whose thickness can be inferred from the grey value to be about $300 \AA$, shall henceforth be called the pancake film. We interpet the shatp boundary belween the pancake libun and the surounding region as at ral space representation of the prewetting line, which is also crossed with increasing distance from the droplet. 
It should be noted that the pancake film forms within the first fourty of milliseconds (two video frames) after the impact and naty well be lormed by direct condensation from the vapor phase rather than spreading of the liquid on the substrate. Some idea of the dynamics of the process can be ganed observing that the two parts (at and b) of Fig. 16 ate only one video frame apart $(20 \mathrm{~ms})$. If the vapur pressure could be assumed to be constant laterally, it would be possible to extract from the width of the boundiry line an estimate of the energy barrier in the effective interface potential hetween the minimum at small thickness and the global minimum at infinily. However; this requires measurements which are closer on equilibrium and shuuld thus be performed on horizontal surfaces to slow dowt the dynamics. These experiments are presently under way.

\subsubsection{Effect of the Kosterlitz-Thouless transition}

When the temperature is raised further, a film as thick as the pancake film atudually extends over the sample, finally covering the whole substrate. At this point, the base pressure in the cell now represents a position in the phase diagrant which is belween the coexistence curve and the prewetting line. When a droplet is now impinging onto the surface, there is no sharp boundary anymore between the bulk region of the droplet and the sturounding region (ct. Fig. 17a). Approakhing the drople rather corre sponds to approaching conexistence starling all pressures above the prewetting line

(a)

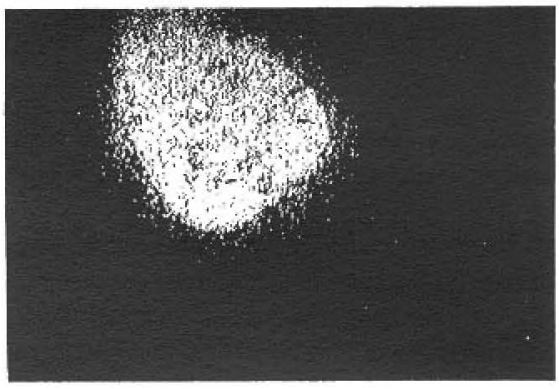

Fig. 17 a) At higher tomperatures, the prewetting line is not anymore being crossed during impact, and the droplet is surrounded by a smooth film profile. b) When the Kosterliz-Thouless line is crossed during impact, a type of puncake film reappears which is of physically different nature than the one shown in Fig. I6.

(b)

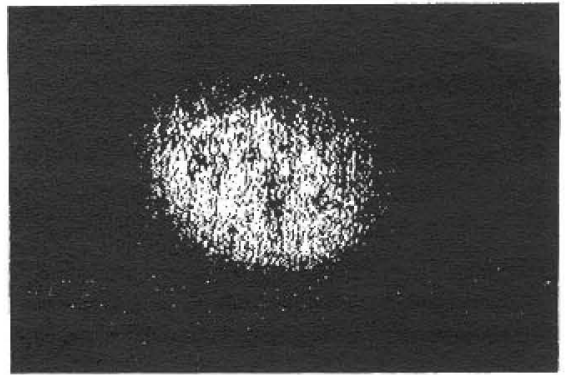


pressure, which results in a smooth divergence of the film thickness towards the droplet.

At even higher temperatures, the reoccurrence of a stepped droplet profile is observed, as shown in Fig. $17 \mathrm{~b}$, which was recorded at $2.1 \mathrm{~K}$. The reason for this to occur is quite obvious when one regards Fig. 12: the excursion in the phase diagram which represents the drop impact crosses the Kosterlitz-Thouless transition line. Hence the outer part of the film is nomal fluid, the inner part (the pancake film) is superfluid. The optical contrast comes about by the fact that the superfluid film is capable of the liquid transport required to replenish the evaporating helium, while the nomal fluid film is not. Thus, the latter readily evaporates, leaving the surface uncovered.

\section{Summary and outlook}

We have demonstrated that many different aspects of wetting may be successfully studied using kryogenic model systems like hydrogen or liquid helium. While the present paper was restricted to two wetting transitions (a continuous and a discontinuous one), it is clear that a large number of phenomena which are also present in more complicated systems are sinilarly accessible to investigation with comparably simple liquids. Similarly to well established studies with binary mixtures of organic liquids [58], it may also be possible to use intertaces between quantum liquids and quantum solids (such as the helium isotopes) as interfaces ol interest. In this case, the problem of surface contamination will be completely avoided, since only cryogenic surfitces remain involved. Systems like these may conne within the reach of exact theoretical tractability.

We gratefully acknowledge stimulating discussions with Sicgfricd Dietrich, Michael Schick, Oscat Vilches, Adrian Wyatt, and Giampaolo Mistura. This work has been supported by the Deutsche Furschungsgemeinschatt (SFB 306 and grant Le 315/18).

\section{References}

11] T. Young, Philos. Trans. Roy. Soc. London 95 (1805) 65

12| S. Dietrich, in 'Phase Transitions and Cratical Phenomena', 12, C. Domb \& J.L. Lebowize (eds.). Acad. Press, L.ondon. 1988

13] M. Schick, in Liquids at Interfaces, Proceedings of the Ies Howhes Summer School, Session XLVIII, J. Charvolin el al. (eds,). Elsevier, Amsicrdam 1990

14] See, for instance, T. Smith, J. Coll. Intl. Sci. 75 (1980) 51

[5] This formula holds as long as the film does not become very thick. For thicker films, retardaton effects change the behaviour; E.S. Salvisky, C.H. Anderson. Phys. Rev, A 7 (1973) 790

[6] I. E. Dzyaloshinskii, E. M. Lifshitz, L. P. Pifaevskii, Adv. Phys, 10 (196i) !65

[7] It should the noted that the wetting material does not need to be in the licuid state. All concepts are equally valid for solid adsorbates

181 Y. L. Clien, C. A. Helm, J.N. Israelachvili, J. Chem. Phys. 95 (1991) 10736

[9] J. Drelich, J.L. Wibur, J.D. Miller, G. M. Whilesides, Langmuir 12 (1996) 1913, and references therein

[10] Strictly speaking, the finite curvature of the droplet renders it being slightly of coexistence, but this is not essential for the discussion and shall not concern us here

1111 V. B. Shenoy, W. F. Saam, Phys. Rev. Lett. 75 (1995) 4086

[12| K. Ragil, J, Meunier, D. Broseta, J.O. Indeket,, D. Bonn, Phys. Rev. Let! 77 (1996) 1532 
(13) 1. M. Tidswell, T. A. Rabcleau, P.S. Pershan, J.P. Folkers, M. V. Baker, G. M. Whitesides, Phys. Rev. B 44 (1991) 10869

[14] G. Reiter, Phys. Rev. I.ell. 68 (1992) 75

[15] P. Lambooy, K.C. Phelatn, O, Hang: G. Kenusch, Phys, Rev, Lett. 76 (1996) 1110

[16] J. Bischof, D. Scherer, S. I lerminghaus, P. Leiderer, Phys. Rev. Lett. 77 (1996) 1536

[17] G. Henn, D.G. Bucknati, M. Stamm, P. Vulhoone, R. Jerome, Macromolecules 29 (1496) 4.305

[18] J. Wilks, 'The Properties of I.iquid and Solid helium', Oxford, 1967

[19] F. Bechatd, P.G. DeGennes, Langmuir 7 (1991) 3216

[20] F. Brochard, J. de Physique (France) II 3 (1993) 21

[21] C. Gay, P.-G. de Gennes, R. Kant, Europhys. Lett. 34 (1996) 58 I

[22] H. Alles, A. V. Babkin, P.J. Hakonen, J.P. Ruutu, J. T. Salojijirvi, J.P. Saramaiki, J. Low Temp. Phys. 102 (1996) 2l, and references therein

[23] A.D. Migone, A. Hoffmann, J. G. Dash, O.E. Vilches, Phys. Rev. B 37 (1988) 5440

[24] N. Bigclow, P.J. Nacher, J. Dupont-Roc, J. Low Temp. Pliys. 89 (1992) 135

|25| see e.g. J.E. Rutledge, P. Taborek, Phys, Rev. Lett. 71 (1993) 263 and references therein

|26| B. Demolder, N. Bigelow, P.J. Nacher, J. Dupont-Roc, J. Low Temp. Phys, 98 (1995) 91

[27] J.R. Sambles. G.W. Bradbery, F. Yang, Contemp. Phys. 32 (1991) 17.3

[28] C.F. Eagen, W. H. Weber, Plys. Rev, B19 (1979) 5068

129] W' Hickel, D. Kump. W. Knoll, Nature 339 (1984) L86

[30] W. Hickel, W. Knoll, Acta Metall. 37 (1989) 2141

131| S. Herminghaus, P. I eiderer, Appl. Phys. L ett. 54 (1989) 96)

132| J. Krim, J.G. Dish, J. Suzanne, Phys. Rev. Lelt. 52 (1984) 640

$133\}$ A.D. Migone, J.G. Dish, M. Schick, O. F. Vilches, Phys. Rev. B 34 (1986) 6322

134 L. Bruschi. G. Toro, M. H.W. Chan, Eutophys, Let. 6 (198s) 541

[35] G. Zimmerli, M.II. W. Chan, Phys. Rev. B 45 (1992) 9347

[36] U. G. Volkmmm, K. Knurr, Phys. Rev. B 47 (1993) 401 I

[37] G. Mistura, H.C. Lee, M.H.W. Chun, J. L.ow Temp, Phys. 96 (1994) 221

[38] J. Krim, J.M. Gay, J. Suzanne, E. Lerner, J. Physique 47 (1986) 1757

139) U. Albrecht, P. Fvers, P. Leiderer, Surf, Sei, 283 (1943) 419

[40] F. Rieutord, R. Simon, R. Conradt, P. Müller-Buschbaum, Europhys. Lett. 37 (1997) 565

[41] Fi. Gittes, M. Schick. Phys. Rev. B 30 (1984) 209

[42] M. A. Grinfeld. Sov. Phys. Dokl. 31 (1986) 831

[43] M. Thiel, A. Willibald, P. Evers, A. Lewehenko, P. Leiderer, S. Balibar, Enrophys. Lett. 20 (1992) 707

[44] U. Albrecht, R. Conradt, S. Herminghas, P. Leiderer, Fiz. Nizk. 'lemp. 22 (1996) 158

$145]$ P. A. Davenport: 'Prospects for Fusion', W. Marshall (ed.) Clarendon Press, Oxford 1983

$146]$ R.S. Craxton, R. L. McCroy, J.M. Sourer, The Scientific American 255 (1986) 60

147| E. W. Otten, Prog. Part. Nucl. Phys. 32 (1994) 153

[48] H. Backe, H. Barth, M. Balzhauser, J. Bonn, B. Degen. L. Fleischmann, P. Leiderer, R. J. Morne, E. W. Otten, S. Schneller, M. Przyrembel, C. Weinheimer, Prog. Part. Nucl. Phys. 32 (1994) 173

[49] E. W. Otten, Nucl. Phys. B Prog. Suppl. 38 (1995) 26

[50] R. N. J. Conradt, U. Albrecht, S. Herminghaus, P. Leiderer, Physica B 194-196 (1994) 679

[51] R. N.J. Conrad,, Thesis University of Konstanz, 1996

[52] II. Raether, 'Surface Plasmons'. Springer Tracts in Modem Physics 111. Springer. Berlin 1988

153) E. Cheng, M.W. Cole, W.F. Saam, J. Treiner, Phys. Rev. I.en. 67 (1991) 1007

[54] J.E. Rutledge, P. Taborek, Phys. Rev. Letl. 69 (1992) 937

[55] J.E. Rutledge, P. Taborek, I. I.ow Temp. Phys. 95 (1994) 405

|56| A.F.G. Wyatt, J. Klier, P. Stefanyi, Phys, Rev, Lett. 74 (1095) 1151

I57] E. Cheng. M.W. Colc, W. F. Sam, J. 'Treiner, J. Low Tump. I'hys, 89 (1992) 739

[58] D. Bonn, H. Kelliy, (i. H. Wegdam, J. Chem. Phys 99 (1993) 7l15, atuf references therein 